\title{
Fine Tuned VGG19 Convolutional Neural Network Architecture for Diabetic Retinopathy Diagnosis
}

\author{
Vijayan $\mathrm{T}^{[1]}$, Sangeetha $\mathrm{M}^{[2]}$, A. Kumaravel ${ }^{[3]}$, Karthik $\mathrm{B}^{[4]}$ \\ Research scholar $^{[1]}$, Professor ${ }^{[2,3]}$,Associate Professor ${ }^{[4]}$ \\ Electronics and Communication Engineering ${ }^{[1,2,4]}$, School of Computing ${ }^{[3]}$ \\ BIST, Bharath Institute of Higher Education and Research \\ tvij16@gmail.com ${ }^{[1]}$
}

\begin{abstract}
Diabetic Retinopathy (DR) is an eye-related complication experienced by individuals with longstanding diabetes. Usually diagnosed by the healthcare professional by retinal fundus examination during Medical check-ups or mass screenings. Early detection of diabetic retinopathy will avoid vision loss and other issues. The objective of this work is to diagnose the Diabetic Retinopathy from retinal fundus images using deep learning (DL) techniques for better detection accuracy.The proposed fine-tunedVGG19 CNN architecture is performing well with the Kaggle data set and effectively dealing with this multi-class classification problem. The proposed model uses pre-trained weights from image net data which lessens the training time and improves the performance in detecting DR from retinal fundus images in terms of sensitivity, specificity, and accuracy. Deep transfer learning with fine-tuning method implementation was carried out to get the highest test accuracy of $73.60 \%$.
\end{abstract}

Keywords: CNN, Diabetic Retinopathy(DR), Deep Learning, Transfer Learning, VGG19

\section{Introduction}

Globally, Diabetic Retinopathy is the major cause of blindness in diabetic patients. Early diagnostics and proper treatment planning will prevent vision loss and blindness. The people having either type 1 or type 2 diabetics for the past 20 years having high-risk factors for DR [7-10]. The DR has been classified into two major types including Proliferative Diabetic Retinopathy (PDR) and Non-ProliferativeDiabetic Retinopathy (NPDR). NPDR is an early stage of DR, which is sub-classified into Mild, Moderate, and Severe stages. PDR is a severe condition where the new blood vessels will start to grow in the inner side of the retina and may spread to the jelly-like portion present inside the eyeballs called the vitreous humor.

Retinal fundus images playa virtual role in early identification for treatment planning in DR stages. Deep learning, the subset of Artificial Intelligence(AI) and Machine Learning (ML) getting a lot of attention for image classification, especially now a day's deep convolutional neural network $[1,3]$ used in remote diagnosis of raw medical images for more accurate prediction of diabetic retinopathy like diseases.

Feng Li et al (2019) [2] proposed a deep transfer learning approach using theInception-v3 network for automatic detectionDR in retinal fundus images and achieved very good accuracy with a higher value of sensitivity and specificity inthe classification of DR images.SehrishQummaret al. (2019) [4] proposedEnsemble Model consists of five CNN architectures including the popular Resnet50, Inceptionv3, Xception, Dense121, and Dense169 architectures to achieve the desired features and higher classification accuracy.Bhavana Sosale et al(2020)[5] proposed an artificial Intelligence (AI) algorithm in the diagnosis of DR using smartphone photographed nonmydriatic (NM) retinal images. This AI system is capable to work without an internet connection and produces instant reports on a smartphone. Stuart Keel et al (2018) [6]proposed referable Diabetic Retinopathy and Glaucomadisease diagnosis based on convolutional neural networks with two authenticated deep-learning models[11].

The objective of this proposed work is to use a Deep Learning algorithm for identifying the Diabetic retinopathy condition from the fundus digital images and it varies from the previous works by suggesting yet another novel framework. The proposed transfer learning technique applied to the VGG 19 network was adopted for the identification of DR or No DR present with different classes in retinal fundus photography.

The rest of the paper content is organized as follows. Section II gives some details on the Transfer learning with VGG19 fine-tuning methodology and Statistical Analysis of Performance. Section III presents the data description and preparation for the training and testing of the classification model. Section IV contains the experimental results. Finally, the research findings and conclusions drawn from this research work are discussed in section $\mathrm{V}$. 


\section{MATERIALS \&METHODS}

The terms/materials used for this experiment are described for the sake of improving the readability for the proposed framework with clarity. Moreover, the rationale behind the classifiers and their representation models are discussed in this section.

\subsection{Transfer learning:}

Transfer learning (TL) is a very popular approach in Deep learning and Machine learning models, this technique first trains a base network on a base dataset for specific task and similarly re-purposed on a secondary related task [12-14]. The benefits of the transfer learning method are to reduce the training time and produce better prediction accuracy innetwork performance. Most of the TL models trained in the image Net database, it contains 1.2 million images with 1000 categories.

\subsection{VGG19:}

Visual Geometry Group 19 (VGG 19) is a powerfulConvolutional Neural Network architectureintroduced by the University of Oxford in 2014. It bagged the 1st runner-upin the Image classification challenge, "ImageNet Large Scale Visual Recognition Competition2014". The VGG 19 architecture is characterized by its pyramidal shape, bottom layers which are closer to the image are wide, whereas the apex layers are deep.VGG 19 consists of 16 convolutional layers (with 5 pooling layers) and 3 fully-connected layers, this network pre-trained with more than 10 lakh images from the ImageNet database.The pre-trained network can classify images into 1000 object categories. this network learned rich feature representations for a wide range of images. The network has a standard image input size of 224 X 224, kernels of ( 3 X 3) size with a stride size of 1-pixel, max pooling was performed over a 2 X 21 window size with stride and the final layer is an activation layer usesSoftMax activation function.

\subsection{Fine tuning:}

The fine-tuning process involves the following steps, (i) unfreezing the last three fully connected layers (ii) adding 2 more FC layers to convert the existing VGG19 model for diabetic retinopathy application. 1000 output classes of the Conventional VGG19 model were reduced to 5 classes representing DR stages due to the fine-tuning process. Whereas trained weights before the FC layersare unchanged. The tuning process increasesthe performance accuracy and ROC values for transfer learning network[15].

\subsection{Statistical Analysis of Performance:}

To estimate the performance of our model in multiclass classification tasks we use metrics like accuracy, sensitivity and specificity[16-17].

o True Positive (TP) represents correctly predicted instances.

o False Positive (FP) represents incorrectly predicted disease instances.

o True Negative (TN) represents correctly predicted no- disease instances.

o False Negative (FN) represents incorrectly predicted no- disease instances

i. Sensitivity is defined as the model's capability to detect the disease correctly and mentioned as

$$
\text { Sensitivity }=\mathrm{TP} /(\mathrm{TP}+\mathrm{FN})
$$

ii. Specificity denotes the model's ability to detect the disease correctly and represented as,

$$
\text { Specificity }=\mathrm{TN} /(\mathrm{TN}+\mathrm{FP})
$$

iii. Accuracy is the ratio of the number of correct predictions to the total number of input samples and denoted as,

$$
\text { Accuracy }=\mathrm{TP}+\mathrm{TN} /(\mathrm{TP}+\mathrm{FN}+\mathrm{TN}+\mathrm{FP})
$$

\section{Data description and preparation}

Kaggle [18] public dataset is used for our experiment to make analysis and identification of Diabetic Retinopathy. Kaggle database provided a large set of high-resolution retina images taken under a variety of spatial conditions. The Left and right field with two different angles of prediction is provided for every subject. Images are labeled with a subject id as well as either left or right (e.g. 1_left.jpeg is the left eye of patient id 1).

This Kaggle dataset contains 35126 color fundus images, among which the first 5000 retinal samples are used for Deep Transfer learning to identify the occurrences of DR, 4000 retinal samples for training, and 1000 retinal samples for testing based on Deep transfer learning technology. It contains the images from five different classes based on the severity of diabetic retinopathy (DR).These images are labeled by an experienced pathologist according to a scale from 0 to 4 associated to 'No_DR', 'Mild', 'Moderate', 'Proliferative DR'. and 'Severe 'respectively as the class labels for the image data records and their distribution is shown in the pie chart as in the Figure 1Before training the model, the pre-processing step is very essential for improving the accuracy rate further. Kaggle public dataset of fundus images contains different dimensions for each image and we resize each input image size to 224X224 pixel resolution, which is the default size for a VGG19 model. 
Table1: Class distribution and description for DR Image dataset (5000 Samples)

\begin{tabular}{|c|c|c|c|}
\hline S. No & Diabetic Retinopathy Types & Instances Count & Class Descriptionof DR \\
\hline 1 & No_DR & 3657 & DR not present \\
\hline 2 & Mild & 354 & DR Mildly present \\
\hline 3 & Moderate & 788 & DR Moderately present \\
\hline 4 & Proliferative DR & 108 & Multiple rapid growths of DR \\
\hline 5 & Severe & 93 & Extremely high presence of DR \\
\hline
\end{tabular}

Table 1 describes the distribution of five class values with their instances count as shown in Figure 1 . The classes are No_DR, Mild, and Moderate, dominate the other two classes namely Proliferative DR and severe.

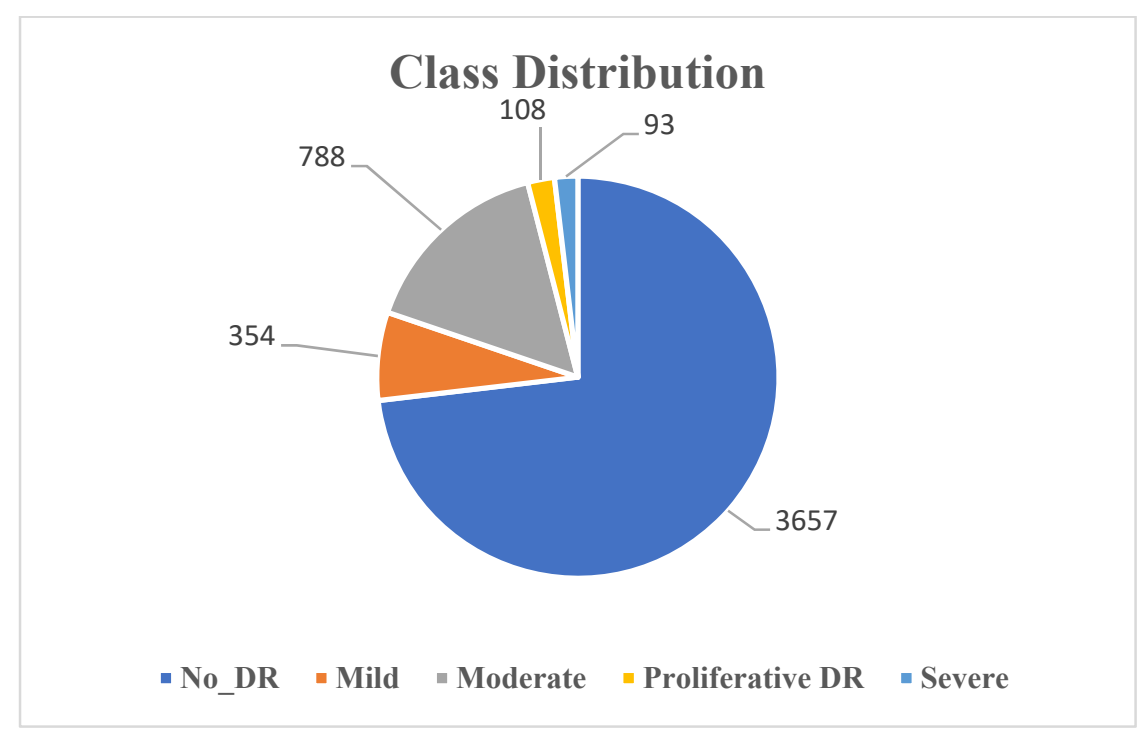

Fig 1: Class distribution for Diabetic Retinopathy

\section{Experiment and Results}

In this section, we have proposed the VGG19 model framework shown in fig 2. Experimental results of the VGG19 fine-tuned Model for 10 epochs shown in Table 2, Graphical plotting of Training accuracy Vs Test accuracy, and Training Loss Vs Test Loss are shown in fig 3 to Fig 4.

\subsection{Proposed Method:}

The proposed work uses a deep transfer learning technique with fine-tuned VGG19 Convolutional Neural Network for classifying the digital retinal fundus image in five classes, which includes No DR, DR, Mild, Moderate, severe, and proliferative DR. The schematic diagram of theproposed architecture is shown in Figure 2. 


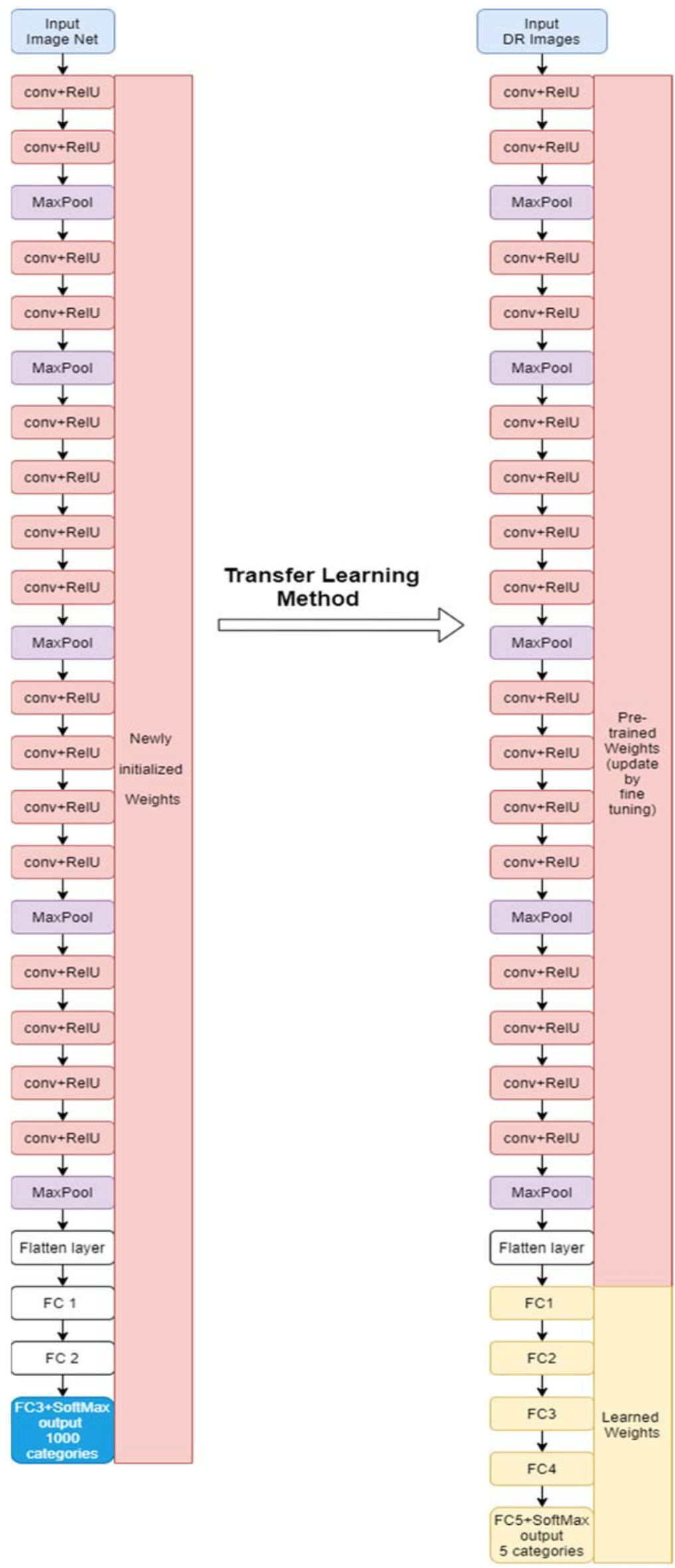

Fig 2:Schematicdiagram of deep transfer learning approach with VGG19 network. 
We transferredthe VGG19 network for categorizing the retinal fundus images using a fine-tuning technique. First, the weights of the convolutional layers were initialized with the pre-trained weights, which was updated during the training process with the ImageNet dataset. This technique will improve the speed of the training process and reduce redundancy. This approach reduces training time and achieves higher accuracy in classification. The last fully connected layers of the VGG19 network architecture are initialized with randomly chosen weights for each neuron to shift the discriminative feature space suitable for retinal fundus photographs rather than ImageNet Data for better learning. The Convolutional Layer and max-pooling layers of the traditional VGG19 are frozen to avoid the update of pre-trained weights. As a part of transfer learning, the last three layers of the network are unfrozen. The flow chartshown in Figure 4 describes the process of the fine-tuning performedon the VGG19 architecture. We add the first Dense layer with Relu activation function, the second Layer includes Drop out of 0.5, and the final dense layer with the SoftMax activation function used,the output layer is modified to classify the images in 1 out of 5 categories, whereas the original architecture consisting of ImageNet database dense layer is capable of categorizing the sample into 1 of the 1000 classes.

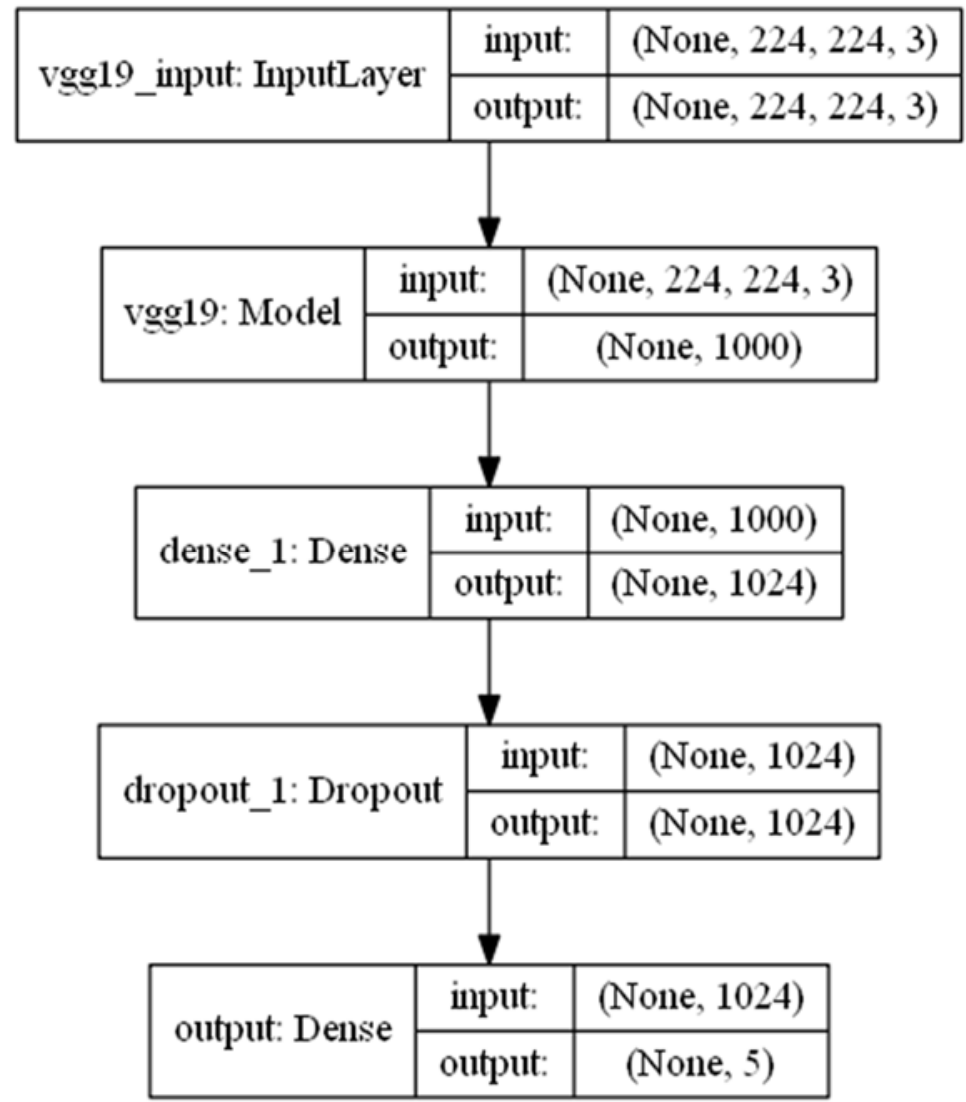

Fig 3:Schematicdiagram of fine-tuning Model

\subsection{Performance Results:}

In this section, we discussed Deep transfer learning Results of fine-tuned VGG19 network. It was trained and tested on a personal computer (PC) with Windows 10, Anaconda Navigator, Jupyter notebook 6.0.3. The hardware configuration of PC,6th generation intel core i7-6500U processor,12GB RAM, NVIDIA GeForce 940M discrete graphics, 1TB hard drive. The proposed model input image size 224x224(default size of VGG19), batch size $=32$, number of classes $=5$ and epochs $=10$. This model uses $80 \%$ samples for training and $20 \%$ samples for testing purpose and for the training process Adam optimizer is selected, categorical cross-entropy loss function are used for improving accuracy level. Table 2 describes the final VGG19 Deep transfer learning running the output of 10 iterations. 
Table 2: Experimental results of VGG19Fine-tuned Model for 10 epochs

\begin{tabular}{|c|c|c|c|c|}
\hline Epoch & Training Loss & Training Accuracy (\%) & Test Loss & $\begin{array}{c}\text { Test } \\
\text { Accuracy (\%) }\end{array}$ \\
\hline 1 & 0.9065 & 72.83 & 0.9872 & 73.60 \\
\hline 2 & 0.8780 & 73.03 & 0.8833 & 73.60 \\
\hline 3 & 0.8769 & 73.03 & 0.4704 & 73.60 \\
\hline 4 & 0.8784 & 73.03 & 1.2427 & 73.60 \\
\hline 5 & 0.8772 & 73.03 & 0.4747 & 73.60 \\
\hline 6 & 0.8773 & 73.03 & 1.1514 & 73.60 \\
\hline 7 & 0.8757 & 73.03 & 0.5002 & 73.60 \\
\hline 8 & 0.8772 & 73.03 & 0.5281 & 73.60 \\
\hline 9 & 0.8793 & 73.03 & 0.3646 & 73.60 \\
\hline 10 & 0.8771 & 73.03 & 0.8012 & 73.60 \\
\hline
\end{tabular}

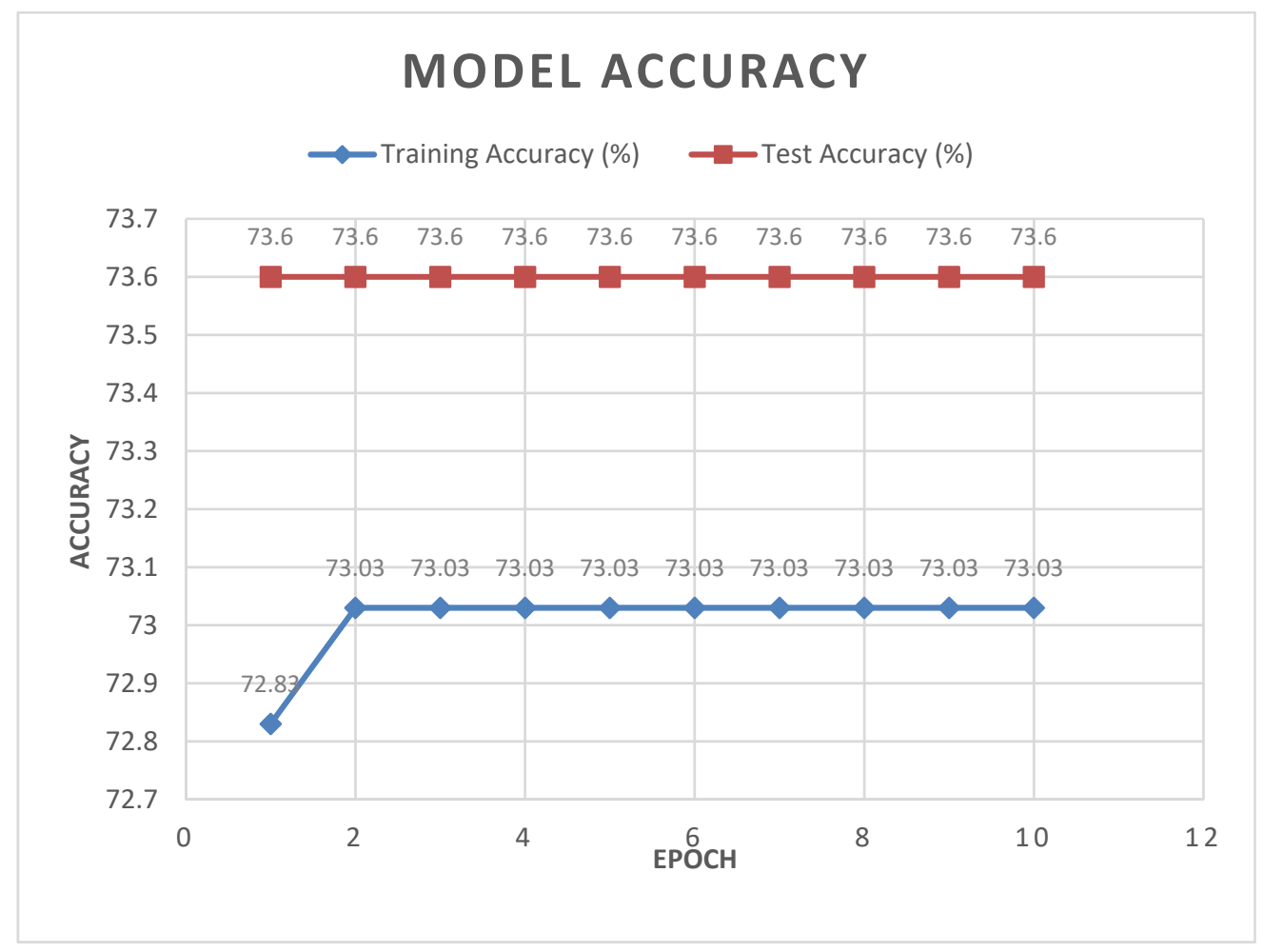

Fig 3: Training accuracy Vs Test accuracy (VGG19 Fine-tuned Model)

Fig 3 clearly describes the graphical representation of 1 to 10 iterations of obtained accuracy level for the training and testing model. We observe that the highest training accuracy $73.03 \%$ and testing accuracy $73.60 \%$ for 10 epochs. Moreover, it reveals not many significant differences in performances either at training or training. 


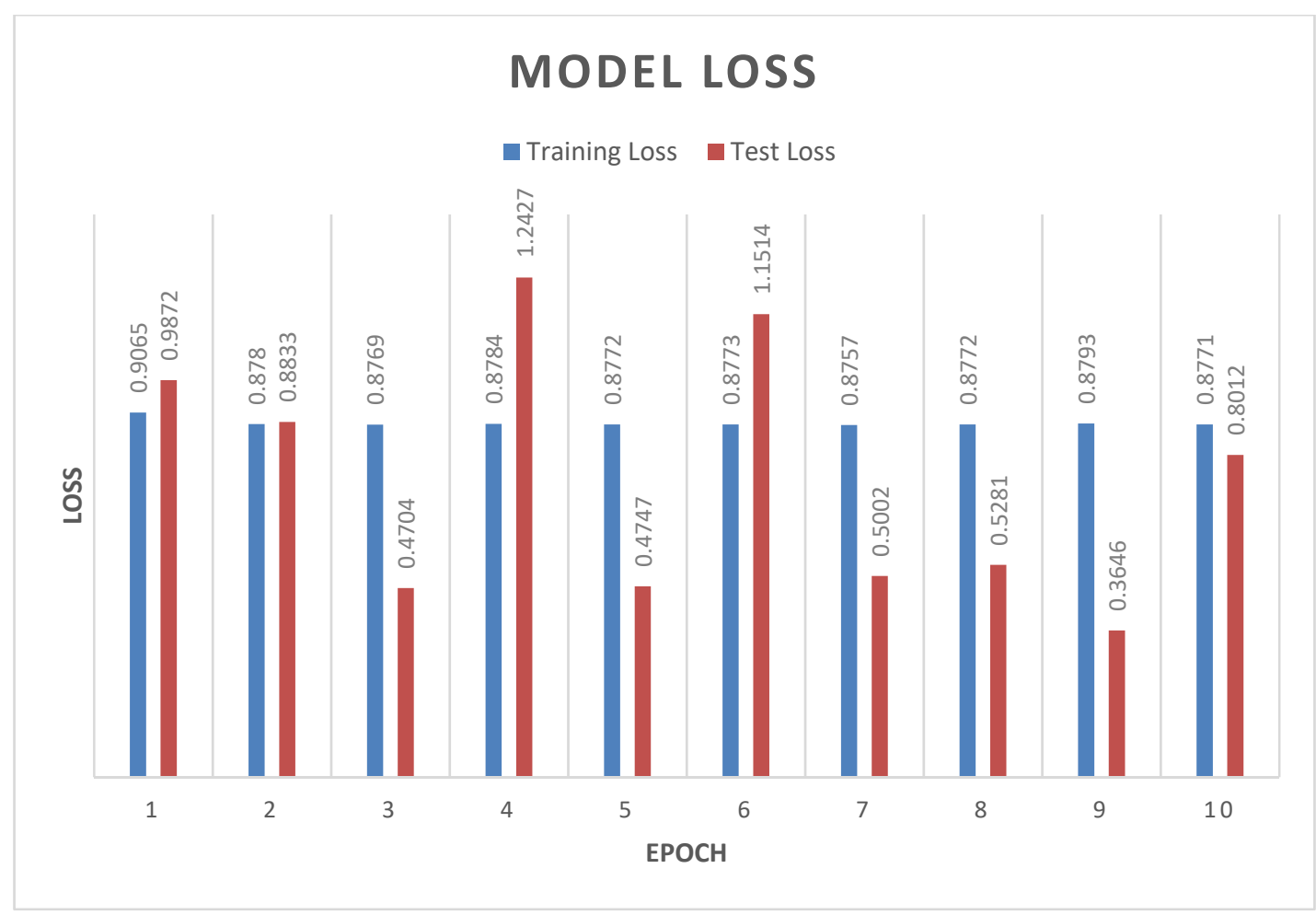

Fig 4: Training Loss Vs Test Loss (VGG19 Fine-tuned Model)

Figure 4 clearly describes the graphical representation of 1 to 10 iterations of obtained loss function for the training and testing model. At the $10^{\text {th }}$ epoch, we obtain 0.8771 for training loss and 0.8012 for test loss.

\section{CONCLUSION}

The proposed novel deep transfer learning approach with the VGG19 network was very efficient for DR multiclass retinal fundus image classification and detection. The optimal accuracy with maximum performance was finally found to be $73.60 \%$ in the testing and $73.03 \%$ for training and the loss occurrence at both training and testing happens to be very less. The transfer learning technique applied on VGG19 CNN architecture yields higher accuracy for multi-class classification of DR in retinal fundus digital images and also presents pre-trained weights so that the training time is very less and also the test images are class-wise identified faster.

\section{REFERENCES:}

[1] C. Mahiba, and A. Jayachandran: Severity analysis of diabetic retinopathy in retinal images using hybrid structure descriptor and modified CNNs, Measurement, Elsevier 135 (2019) pp.762-767.

[2] Feng Li \& Zheng Liu et al: Automatic Detection of Diabetic Retinopathy in Retinal Fundus Photographs Based on Deep Learning Algorithm, tvst, vol 8, No 6, 2019, pp 1-13.

[3] Filippo Arcadu and FethallahBenmansour et al: Deep learning algorithm predicts diabetic retinopathy progression in individual patients, npj Digital Medicine (2019), pp 1-9.

[4] SEHRISH QUMMAR \& FIAZ GUL KHAN et al: A Deep Learning Ensemble Approach for Diabetic Retinopathy Detection, IEEE Access, vol 7, oct 29, 2019, pp150530- 150539 .

[5] BhavanaSosale and Sosale Ramachandra Aravind et al: Simple, Mobile-based Artificial Intelligence Algorithm in the detection of Diabetic Retinopathy (SMART) study, BMJ Open Diab Res Care, 2020, pp 1-6.

[6] Stuart Keel and JinrongWu, et al:Visualizing Deep Learning Models for the Detection of Referable Diabetic Retinopathy and Glaucoma,JAMA,Dec 20,2018,pp E1-E5.

[7] Rahul Kumar Chaurasiya, MohdImroze Khan et al: BPSO-Based Feature Selection for Precise Class Labeling of Diabetic Retinopathy Images, Advanced Engineering Optimization Through Intelligent Techniques, Springer, 10 July 2019,pp 253-264

[8] R. Karthikeyan \& P. Alli et al: Feature Selection and Parameters Optimization of Support Vector Machines Based on Hybrid Glowworm Swarm Optimization for Classification of Diabetic Retinopathy,Journal of Medical Systems, volume 42, Article number: 195 (2018)

[9] R. Valarmathi S. Saravanan et al: Exudate characterization to diagnose diabetic retinopathy using generalized method, Journal of Ambient Intelligence and Humanized Computing, 19 Dec 2019.

[10] T. Jemima Jebaseeli, C. Anand Deva Durai, and J. Dinesh Peter: Segmentation of retinal blood vessels from ophthalmologic Diabetic Retinopathy images, Elsevier, Computers and Electrical Engineering, 73 (2019), pp.245-258.

[11] Pedro Costa, Adrian Galdran and AsimSmailagic: A Weakly-Supervised Framework for Interpretable Diabetic Retinopathy Detection on Retinal Images, Special Section on Advanced Signal Processing Methods in Medical Imaging, IEEE Access, vol 6, April 2018, pp.18747-18758.

[12] B. Wu, W. Zhu, F. Shi, S. Zhu, and X. Chen: Automatic detection of microaneurysms in retinal fundus images, Computerized Medical Imaging and Graphics, vol. 55, pp. 106-112, 2017.

[13] Toan Bui, NoppadolManeerat et al: Detection of Cotton Wool for Diabetic Retinopathy Analysis using Neural Network, 2017 IEEE 10th International Workshop on Computational Intelligence and Applications, November 11-12, 2017, Hiroshima, Japan.

[14] Varun Gulshan, Lily Peng et al: Development and Validation of a Deep Learning Algorithm for Detection of Diabetic Retinopathy in Retinal Fundus Photographs, JAMA, Published online November 29, 2016. 
[15] Kranthi Kumar Palatalise and BhavaniSambaturu: Automatic Diabetic Retinopathy Detection Using Digital Image Processing, International Conference on Communication and Signal Processing, April 3-5, 2018, PP-0072-0076, India.

[16] Shailesh Kumar and Basant Kumar: Diabetic Retinopathy Detection by Extracting Area and Number of Microaneurysm from Colour Fundus Image,2018 5th International Conference on Signal Processing and Integrated Networks (SPIN), pp.359-364.

[17] Daniel ShuWei Ting, Alfred Gan et al: Development and Validation of a Deep Learning System for Diabetic Retinopathy and Related Eye Diseases Using Retinal Images from Multi-ethnic Populations with Diabetes, JAMA, Volume 318, No 22, December12, pp. 22112223,2017.

[18] Kaggle: https://www.kaggle.com/ 